\title{
The threat of chikungunya in Oceania
}

\author{
Paul Horwood, a Grace Bande, a Rosheila Dagina, b Laurent Guillaumot, c John Aaskov ${ }^{d}$ and Boris Pavline \\ Correspondence to Paul Horwood (e-mail: paul.horwood@pngimr.org.pg).
}

$\mathrm{T}$ he Oceania region, which includes Australia, New Zealand, Papua New Guinea and the islands of the tropical Pacific Ocean, has historically been free from chikungunya. However, the 2011 outbreak in New Caledonia and the ongoing outbreak in Papua New Guinea have highlighted the risk to other communities in Oceania where there are competent mosquito vectors and permissive social factors and environmental conditions. In this article we discuss the threat to this region that is posed by the recent evolution of the E1:A226V mutant strains of chikungunya virus (CHIKV).

Chikungunya is a mosquito-borne disease caused by infection with $\mathrm{CHIKV}$, an alphavirus from the Togaviridae family. The clinical characteristics of chikungunya include acute onset of fever which may last up to two weeks and painful, potentially debilitating, polyarthritis in adults which may last for up to a year following infection. Chikungunya was first recognized in Africa in the 1950s, principally, as polyarthritis in adults. ${ }^{1}$ Other symptoms, reported during the large outbreak on Réunion Island in 2005-2006, included maculopapular rash on the trunk and limbs, headache, nausea, vomiting, diarrhoea and fatigue. $^{2}$

There are three distinct genotypes of CHIKV: (1) Asian, (2) Eastern/Central/Southern African (ECSA), and (3) Western African. The ECSA genotype has been the dominant strain throughout Asia and the islands and countries in the Indian Ocean over the last decade. This genotype gained dominance in 2004 and 2005 when it was introduced from Kenya into the Indian Ocean islands of Comoros, Réunion, Seychelles, Mauritius and Mayotte where it was associated with an outbreak involving hundreds of thousands of reported cases. $^{3}$ On the island of Réunion, it was estimated that more than $30 \%$ of the 770000 inhabitants were infected by $\mathrm{CHIKV}^{2}$ In 2005, an epidemic of chikungunya began in India

which resulted in more than 1.39 million suspected cases by $2011 .{ }^{4}$ The ECSA genotype of CHIKV also spread to other Asian countries including Sri Lanka, Malaysia, Singapore, Thailand, Indonesia, China and Myanmar. ${ }^{3,5}$

Previous outbreaks of CHIKV infection have been associated with the mosquito vector Aedes aegypti, which is also the vector of yellow fever and dengue viruses. However, Aedes albopictus has been the principal mosquito vector during many of the recent outbreaks of chikungunya associated with ECSA strains. ${ }^{6}$ Analysis of CHIKV from the explosive outbreaks in Réunion and India revealed that the ECSA strains had acquired a point mutation resulting in a change from alanine to valine at position 226 in the E1 glycoprotein which enhanced the transmissibility of CHIKV in Aedes albopictus. ${ }^{7}$ Subsequent studies demonstrated that amino acid changes in the E2 glycoprotein had a strong modulating effect on the E1:A226V change. ${ }^{8}$

Until the outbreak of chikungunya in New Caledonia from February to June 2011, which was caused by Asianlineage CHIKV rather than the E1:A226V ESCA lineage, ${ }^{9}$ Oceania had been free from chikungunya. During this outbreak, only 33 cases were detected, attributed to the onset of the cold season and the comprehensive control measures implemented after the diagnosis of the first cases.

In June 2012, an outbreak of fever and arthritis was detected in Vanimo, Papua New Guinea. Subsequent investigations showed that the outbreak was caused by an ECSA strain of CHIKV which harboured the E1:A226V mutation. During the Vanimo outbreak more than 1500 suspected cases of chikungunya were reported through passive surveillance. ${ }^{10}$ The vector in this outbreak was suspected to be Aedes albopictus due to the high density of this mosquito species in the area.

\footnotetext{
Papua New Guinea Institute of Medical Research, Goroka, Papua New Guinea.

Papua New Guinea National Department of Health, Port Moresby, Papua New Guinea

Institut Pasteur de Nouvelle Calédonie, Noumea, Nouvelle-caledonie.

WHO Collaborating Centre for Arbovirus Reference and Research, Queensland University of Technology, Brisbane, Australia.

World Health Organization, Port Moresby, Papua New Guinea.

Submitted: 10 April 2013; Published: 00 May 2013

doi: 10.5365/wpsar.2013.4.2.003
} 
Figure 1. Recent outbreaks of chikungunya in Oceania and the distribution of Aedes vectors

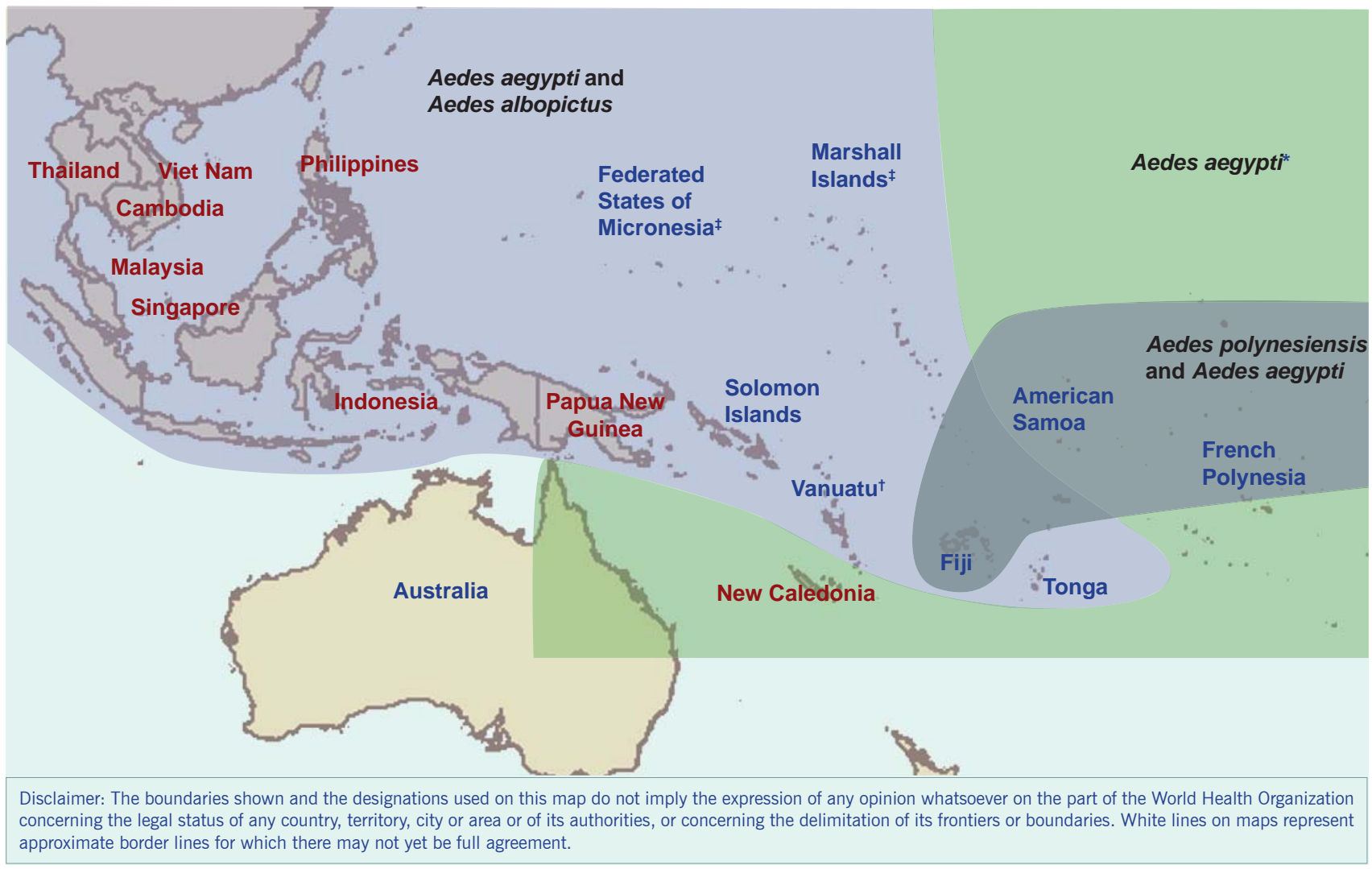

Note: Countries in red had previous chikungunya outbreaks.

Aedes aegypti is found throughout the region except in Futuna and some isolated islands.

The presence of Aedes albopictus has not been officially confirmed in Vanuatu; however, its presence is strongly suspected.

* Aedes albopictus has not been detected in the Marshall Islands or the Federated States of Micronesia; however, its presence is suspected due to the proximity of islands such as Guam and Palau where the vector has been confirmed.

Following this outbreak, chikungunya cases were confirmed by real-time reverse-transcriptase polymerase chain reaction from eight provinces of Papua New Guinea, with another three provinces having suspected outbreaks. Interestingly, the outbreak extended to the Highlands Region of Papua New Guinea, which is the first confirmed arboviral outbreak recorded in this region of the country. Although no entomologic surveys have been conducted in the Highlands Region for many years, it has been shown that Aedes mosquitoes are present in abundant numbers. This may have important implications as more than $50 \%$ of the Papua New Guinea population live in the Highlands Region.

In Oceania, a considerable number of endemic mosquitoes belonging to the genus Stegomyia and the "Scutellaris group," such as Aedes polynesiensis in French Polynesia, are recognized as vectors of CHIKV. ${ }^{11,12}$ More importantly, the principal vectors of chikungunya, Aedes aegypti and Aedes albopictus, are both prevalent throughout the region. Aedes aegypti is present in all countries in the Pacific except for New Zealand, Futuna and some small isolated islands. ${ }^{13}$ Aedes albopictus invaded Oceania in the $1960 s^{14}$ and now can be found throughout Papua New Guinea, the Torres Strait region of Australia, Fiji, Solomon Islands, Tonga and probably Vanuatu, ${ }^{13,15}$ thus rendering the human populations of these islands vulnerable to introduction of the epidemic ECSA strains of CHIKV. The explosive outbreak of chikungunya in the Indian Ocean islands and the speed with which the related alphavirus, Ross River virus, swept through the Pacific in 1979 and $1980,{ }^{16}$ is a reminder of the potential impact CHIKV could have in Oceania (Figure 1).

Social, economic and environmental factors all play an important role in the introduction and sustained transmission of arboviral diseases like chikungunya. In developing countries such as Papua New Guinea and many other Pacific island communities, poor living conditions and the abundance of natural and artificial 
mosquito breeding sites can result in the rapid spread of arboviral epidemics. The climatic conditions of Oceania (temperature, humidity) favour year-round mosquito breeding and are unlikely to interrupt the transmission cycle of CHIKV. It is doubtful that any Pacific island community has the human or financial resources to mount a vector control effort that would prevent an outbreak of chikungunya. However, efficient surveillance, targeted vector control (including active community participation for breeding sites elimination) and education in mosquito avoidance measures may provide a cost effective reduction in the burden of disease in the event of an outbreak. A coordinated regional strategy to prevent and respond to vectorborne disease outbreaks in Oceania is urgently needed to mitigate future outbreaks of arboviral diseases such as chikungunya and dengue.

\section{Conflicts of interest}

None declared.

\section{Funding}

None.

\section{References:}

1. Robinson MC. An epidemic of virus disease in Southern Province, Tanganyika Territory, in 1952-53. I. Clinical features. Transactions of the Royal Society of Tropical Medicine and Hygiene, 1955, 49:28-32. doi:10.1016/0035-9203(55)90080-8 pmid: 14373834

2. Borgherini $G$ et al. Outbreak of chikungunya on Reunion Island: early clinical and laboratory features in 157 adult patients. Clinical Infectious Diseases, 2007, 44:1401-1407. doi: 10.1086/517537 pmid:17479933

3. Ng LC, Hapuarachchi HC. Tracing the path of Chikungunya virusevolution and adaptation. Infection, Genetics and Evolution, 2010, 10:876-885. doi:10.1016/j.meegid.2010.07.012 pmid:20654736

4. Kumar NP et al. A226V mutation in virus during the 2007 chikungunya outbreak in Kerala, India. Journal of General
Virology, 2008, 89:1945-1948. doi:10.1099/vir.0.83628-0 pmid:18632966

5. Qiaoli Z et al. Maiden outbreak of chikungunya in Dongguan City, Guangdong Province, China: epidemiological characteristics. PLOS ONE, 2012, 7:e42830. doi:10.1371/journal.pone.0042830 pmid:22916166

6. de Lamballerie $X$ et al. Chikungunya virus adapts to tiger mosquito via evolutionary convergence: a sign of things to come? The Journal of General Virology, 2008, 5:33-36. doi:10.1186/1743-422X-5-33 pmid:18304328

7. Tsetsarkin KA et al. A single mutation in chikungunya virus affects vector specificity and epidemic potential. PLoS Pathogens, 2007, 3:e201. doi:10.1371/journal. ppat.0030201 pmid:18069894

8. Tsetsarkin KA et al. Epistatic roles of E2 glycoprotein mutations in adaption of chikungunya virus to Aedes albopictus and Aedes aegypti mosquitoes. PLoS ONE, 2009, 4:e6835. doi:10.1371/ journal.pone.0006835 pmid:19718263

9. Dupont-Rouzeyrol $\mathrm{M}$ et al. Chikungunya virus and the mosquito vector Aedes aegypti in New Caledonia (South Pacific Region). Vector Borne and Zoonotic Diseases, 2012, 12:1036-1041. doi:10.1089/vbz.2011.0937 pmid:23167500

10. Horwood PF et al. Outbreak of chikungunya virus infection, Vanimo, Papua New Guinea. Emerging Infectious Diseases, 2013 doi:10.3201/eid1909.130130

11. Gilotra SK, Shah KV. Laboratory studies on transmission of Chikungunya virus by mosquitoes. American Journal of Epidemiology, 1967, 86:379-385. pmid:4383438

12. Guillaumot L. Arboviruses and their vectors in the Pacificstatus report. Pacific Health Dialog, 2005, 12:45-52. pmid: 18181493

13. Guillaumot $\mathrm{L}$ et al. Distribution of Aedes albopictus (Diptera, Culicidae) in southwestern Pacific countries, with a first report from the Kingdom of Tonga. Parasites \& Vectors, 2012, 5:247252. doi:10.1186/1756-3305-5-247 pmid:23130961

14. Cooper RD et al. Aedes albopictus (Skuse) (Diptera: Culicidae) in the Western Province of Papua New Guinea and the Threat of its Introduction to Australia. Journal of the Australian Entomological Society, 1994, 33:115-116. doi:10.1111/j.1440-6055.1994. tb00933.x

15. Ritchie SA et al. Discovery of a widespread infestation of Aedes albopictus in the Torres Strait, Australia. Journal of the American Mosquito Control Association, 2006, 22:358-365. doi:10.2987/8756-971X(2006)22[358:DOAWIO]2.0.CO;2 pmid: 17067032

16. Derraik JG et al. Chikungunya virus: a novel and potentially serious threat to New Zealand and the South Pacific islands. The American Journal of Tropical Medicine and Hygiene, 2010, 83:755-759. doi:10.4269/ajtmh.2010.10-0123 pmid:20889861 